\title{
La política pública de seguridad y salud en el trabajo: el caso colombiano
}

\author{
Public Policy for Safety and Health at the Worksite: the Colombian Case \\ A política pública de segurança e saúde no trabalho: o caso colombiano
}

\author{
Sonia Helena Álvarez Torres ${ }^{a}$ \\ Universidad Jorge Tadeo Lozano, Colombia \\ shalvarezt@yahoo.com \\ ORCID: http://orcid.org/0000-0001-6992-4381 \\ Martha Isabel Riaño-Casallas \\ Universidad Nacional de Colombia \\ ORCID: http://orcid.org/0000-0002-9384-1428
}

DOI: https://doi.org/10.11144/Javeriana.rgps17-35.ppss Redalyc: http://www.redalyc.org/articulo.oa?id=54557477015

Fecha de recepción: 16 Agosto 2017

Fecha de aprobación: 20 Abril 2018

Fecha de publicación: 01 Noviembre 2018

\section{Resumen:}

Se espera que el trabajo proporcione riqueza y bienestar al ser humano, pero toda actividad laboral incluye fatalidades, accidentes y enfermedades ocupacionales que afectan la productividad laboral y el desarrollo sostenible. La seguridad y la salud buscan construir una cultura de entornos laborales saludables, trabajo seguro y decente, y para ello el Estado colombiano promueve políticas públicas de seguridad a partir de recomendaciones de organismos internacionales, algunas de ellas enmarcadas en los retos del milenio. El objetivo de esta revisión es presentar como ha sido el desarrollo de la política pública colombiana en seguridad y salud en el trabajo, para lo cual se mostrarán las directrices internacionales postuladas por la OIT y la OMS, su despliegue en estrategias iberoamericanas y la aplicación de la política pública en los programas nacionales de seguridad y salud en el trabajo.

Palabras clave: política pública, salud laboral, políticas públicas de salud ocupacional, programa de prevención de riesgos en el ambiente laboral, riesgos laborales, Colombia.

\begin{abstract}
:
Work is expected to provide the human being with richness and well-being; notwithstanding all working activity implies fatalities, accidents and occupational diseases affecting the work productivity and the sustainable development. Safety and health are pivotal to build a culture of both healthy working environments and safe and respectable work. To accomplish this, the Colombian State is promoting safety public policies based on the recommendations by international agencies, some of them framed in the new millennium challenges. Ae review aims to show how the Colombian public policy for safety and health at the worksite has developed so far. To do so, the international guidelines set by both the ILO and the WHO will be outlined, showing how they are implemented through the Iberian-American strategies and the application of public policy in the national programs for safety and health at the worksite.
\end{abstract}

Keywords: public policy, labor health, occupational health public policies, prevention program for labor hazards, labor hazards, Colombia.

\section{Resumo:}

Espera-se o trabalho fornecer riqueza e bem-estar para o ser humano, mas toda atividade laboral inclui fatalidades, acidentes e doenças ocupacionais que afetam a produtividade laboral e o desenvolvimento sustentável. A segurança e a saúde visam construir uma cultura de ambientes laborais saudáveis, trabalho seguro e decente, e para isso o Estado colombiano promove políticas públicas de segurança a partir de recomendações de organismos internacionais, algumas delas enquadradas nos desafios do milénio. O objetivo desta revisão é apresentar como tem sido o desenvolvimento da política pública colombiana em segurança e saúde no trabalho, para o qual foram mostradas as diretrizes internacionais postuladas pela OIT e pela OMS, seu desfralde em estratégias ibero-americanas e a aplicação da política pública nos programas nacionais de segurança e saúde no trabalho.

Palavras-chave: política pública, saúde laboral, políticas públicas de saúde ocupacional, programa de prevenção de riscos no ambiente laboral, riscos laborais, Colômbia.

\footnotetext{
${ }^{\text {a }}$ Autora de correspondencia: shalvarezt@yahoo.com
} 


\section{Introducción}

Desde siempre, la relación hombre-trabajo ha estado polarizada entre el desarrollo personal, la competitividad empresarial y su impacto socioeconómico, versus el lado negativo de la afectación de la salud, del ambiente y de la vida (1), convirtiéndose en un proceso que ha conducido a múltiples propuestas de intervención, primero individuales y posteriormente de acciones colectivas de protección de la salud en el trabajo; sin embargo, es en la década de 1950 cuando la Organización Internacional del Trabajo (OIT y la Organización Mundial de la Salud (OMS) constituyen un comité conjunto para aunar esfuerzos y postular la medicina del trabajo como "el conjunto de disciplinas que tiene como finalidad fomentar y mantener el más alto grado de bienestar físico, mental y social de los trabajadores en todos los oficios, prevenir todo daño a la salud por el entorno laboral y proteger a los trabajadores de los riesgos del trabajo" (2). En 1959, con ocasión de la promulgación de la Resolución 112 sobre servicios de Medicina del Trabajo se acuña el término salud ocupacional (SO)(3), el cual es ajustado, por ellos mismos, en 1995, indicando que "el énfasis de la salud ocupacional está orientado al mantenimiento y promoción de la salud de los trabajadores y su capacidad de trabajo, mejorar el ambiente de trabajo y el trabajo mismo y al desarrollo de una cultura de trabajo sano y seguro reflejada en los sistemas gerenciales, los principios de participación, las políticas de entrenamiento y la calidad gerencial del compromiso" (4).

En el nuevo milenio, la OIT, consciente de la estrecha relación entre las condiciones y el medio ambiente de trabajo y la salud y la productividad, introduce la noción de seguridad y salud en el trabajo (SST, entendida como "una disciplina que trata de la prevención de las lesiones y enfermedades relacionadas con el trabajo y de la protección y promoción de la salud de los trabajadores. La salud en el trabajo conlleva la promoción y el mantenimiento del más alto grado de salud física y mental y de bienestar de los trabajadores en todas las ocupaciones" (5). "También se deberían tener en cuenta los posibles efectos en las comunidades vecinas y en el medio ambiente general hacia la gestión integradora de la salud en el lugar de trabajo" (6). De manera que el concepto ha venido evolucionando y, aunque asume diversos significados, mantiene sus lineamientos originales de protección a los trabajadores, fomento del bienestar bio-psico-social y prevención de los desenlaces en salud, pero, cada vez más, se amplía a los entornos de trabajo libres de riesgos, al mantenimiento de la capacidad para laborar y al compromiso gerencial como elementos de la productividad empresarial e integrado con la cultura de cuidado a las personas y al ambiente, lo cual conduce a sociedades "capaces de satisfacer las necesidades de la generación presente sin comprometer la capacidad de generaciones futuras", como lo indica el principio rector del desarrollo sostenible mundial (7).

En este orden de ideas, la seguridad y salud en el trabajo (SST), concepto al que se hará referencia de aquí en adelante, está inmersa en las necesidades empresariales, comunitarias y globales, por lo que ha reclamado reconocimiento a partir de lineamientos mundiales que, a su vez, han instado a los gobiernos a plantear políticas públicas nacionales. Para comprender el significado de la política pública de SST, es necesario precisar el término política en castellano, el cual está condicionado por tres de sus acepciones en inglés, a saber: polity, politics, y policy. "política concebida como el gobierno y las formas de gobierno de las sociedades humanas es polity, politica como la actividad de organización y lucha por el control del poder es politics y política a través de propósitos e intervenciones de las autoridades públicas en beneficio de la sociedad es policy" (8). La policy o la política pública es política y es pública, ya que corresponde a la intervención del Estado que diagnostica una situación, la ingresa a la agenda gubernamental, formula, desarrolla y ajusta (9). Desde la relación Estado-sociedad la política pública se entiende como "el proceso integrador de decisiones, acciones, inacciones, acuerdos e instrumentos, adelantado por autoridades públicas con la participación eventual de los particulares, y encaminado a solucionar o prevenir una situación definida como problemática. La política pública hace parte de un ambiente determinado del cual se nutre y al cual pretende modificar o mantener" (10). El concepto de política pública permite "enlazar bajo un mismo término la producción normativa de las instituciones públicas — planes, leyes, fallos jurídicos, etc. — con las 
actividades realizadas tanto por los actores políticos y sociales como por las autoridades públicas" para elaborar, mantener y ajustar las decisiones y sus efectos en la sociedad (11).

La política pública de SST delineada internacionalmente aparece en 1981 cuando la OIT adopta el Convenio 155 sobre seguridad y salud de los trabajadores, que entra en vigor en 1983. En este se presenta de manera formal una política nacional de SST para prevenir los desenlaces de salud no deseados que sean consecuencia de las labores en el entorno de trabajo (12). Como parte de esta política, la OIT pide que se garantice el registro y notificación de los accidentes de trabajo y enfermedades laborales (ATEL, y esto queda manifiesto en el Protocolo 155 del 2002 (13). A comienzos del siglo XXI, reconociendo el impacto negativo de los ATEL sobre el bienestar de las personas, la productividad y el desarrollo socioeconómico, y considerando que la promoción de la SST forma parte del programa Trabajo Decente para Todos, la OIT lanza su Estrategia Global en materia de SST y presenta el Convenio 187 sobre el marco promocional para seguridad y salud en el trabajo que desarrolla el enfoque estratégico mediante la creación de una política, un sistema, el perfil y los programas nacionales en SST.

Por otra parte, se destaca la importancia de desarrollar una "cultura nacional de prevención en materia de SST", aquella en la que el derecho a un medio ambiente de trabajo seguro y saludable se respeta en todos los niveles, en la que el Gobierno, los empleadores y los trabajadores participan activamente en iniciativas destinadas a garantizar un medio ambiente de trabajo seguro y saludable mediante un sistema de derechos, responsabilidades y deberes bien definidos, y en la que se concede la máxima prioridad al principio de prevención (14). Lo anterior se complementa con la Recomendación 197, la cual plantea que la política nacional debería tener en cuenta los derechos, las obligaciones y las responsabilidades pertinentes de los gobiernos, los empleadores y los trabajadores y que el programa nacional se sustente en los principios de evaluación y gestión de peligros y riesgos (15).

De acuerdo con lo anterior, una política nacional de $S S T$ es el marco de acción específico adoptado por un Gobierno, en consulta con sus interlocutores sociales, para definir su mandato en materia de SST (16). Esta se plantea con el fin de garantizar que se minimicen, en la medida en que sea razonable y factible, los riesgos del trabajo, que se prevengan los ATEL y se favorezca un medio ambiente de trabajo propicio para la salud de los trabajadores. Cada país, habida cuenta de sus condiciones y prácticas, debe formular, implementar y examinar periódicamente dicha política con el fin de ajustarla según sea el caso. Para esto, hay que designar funciones a las autoridades públicas e involucrar a los actores del sistema nacional, siguiendo ciertas líneas de acción, garantizando el cumplimiento de las exigencias legales y promoviendo la cultura nacional de prevención en materia de SST $(16,17)$.

La política nacional se despliega a través de un Sistema Nacional de SST, que es la infraestructura legal e institucional que permite articular los elementos y las funciones para que actúen como un todo capaz de lograr el mandato (18). Los elementos principales de un sistema nacional de SST incluyen: autoridades competentes, órgano consultivo tripartito, legislación, procesos de vigilancia, mecanismos de apoyo a las empresas y servicios de educación y de administración de la información. Como parte del marco promocional de la SST, los sistemas nacionales permiten a los países gestionar eficazmente sus recursos para cumplir con sus funciones y lograr sus propósitos (19).

El perfil de SST es el primer paso en el proceso de elaboración de un programa nacional, ya que resume la situación de SST del país, analiza el sistema desde la identificación de convenios internacionales y la legislación, el marco institucional, los recursos, así como los ATEL (20). Se usa como base para identificar las prioridades de acción; sirve para sensibilizar e instar el apoyo de la ciudadanía al conocer la situación actual del país en materia de SST; pero, sobre todo, es una herramienta de gestión que, a través de su actualización periódica, sirve para medir los progresos en el despliegue de la política (21).

Por otra parte, el conjunto de intervenciones priorizadas, con objetivos y metas en un tiempo determinado e indicadores para evaluar su avance configuran un Programa Nacional de SST (16). Este puede recibir otros nombres, como Estrategia, Plan Estratégico, Plan de Acción, Plan Nacional, pero 
en general con características afines al propósito al que sirven (22). Un programa nacional de SST debe promover el desarrollo de una cultura de prevención en materia de seguridad y salud; además de contribuir a la protección de los trabajadores mediante la eliminación o minimización de los riesgos relacionados con el trabajo, para prevenir lesiones, enfermedades y muertes. Puede ser apoyado, cuando aplique, por otros programas nacionales complementarios y planes que asistan el logro de un ambiente de trabajo seguro y saludable (22). Los programas nacionales se postulan sobre la base de necesidades, riesgos y logros de cada nación e imponen la participación de instituciones gubernamentales competentes, empleadores y trabajadores. Los programas están dirigidos a movilizar las acciones de promoción de la salud, prevención de los accidentes y enfermedades laborales y protección de los trabajadores; propiciar las redes de información, la educación pluridisciplinaria, la investigación en SST y reforzar los modelos de inspección, vigilancia y control nacionales. Es necesario hacer seguimiento a sus indicadores en términos de progresos y de resultados para alimentar las acciones futuras en SST (23).

Las políticas universales de SST, como se ha mencionado, han sido lideradas por dos organismos del Sistema de Naciones Unidas, la OIT, agencia de composición tripartita, en tanto que reúne a gobiernos, empleadores y trabajadores, (24), y la OMS, autoridad directiva y coordinadora en asuntos de sanidad internacional (25). Ambas entidades, con perspectivas específicas y actividades articuladas, han venido desarrollando innumerables iniciativas en busca de la gestión sistemática de los riesgos del trabajo, de forma tal que se logren resultados positivos, no solo en la cultura de prevención, sino con entornos laborales saludables y seguros (26) para que "el trabajo decente signifique también trabajo seguro" (27). Sus intenciones han sido acogidas mundialmente e Iberoamérica ha hecho acopio de estas a través de estrategias regionales que se constituyen en instrumentos para establecer el marco general de las políticas públicas nacionales de seguridad y salud en el trabajo (28).

En definitiva, la política pública en SST no solo es una apuesta país para materializar la acción del Estado, sino el instrumento que la seguridad y la salud en el trabajo urgen como respuesta específica, práctica y eficaz para gestionar los riesgos e impactar verdaderamente la morbimortalidad laboral. Solo así, cada país podrá contribuir a la construcción de una cultura de prevención de enfermedades y accidentes de trabajo en entornos laborales saludables, como componente esencial del trabajo decente, y este, junto con el crecimiento económico, concretarse en los objetivos ecuménicos de desarrollo sostenible. De allí y en consideración a todo lo anterior, el objetivo del presente artículo es analizar cómo ha sido el desarrollo de la política pública de SST en Colombia, para lo cual se discutirán los lineamientos globales en materia de seguridad y salud en el trabajo, sus implicaciones en las estrategias regionales y la aplicación en la política pública de SST colombiana.

\section{La OIT y la OMS, mentores de los lineamientos de seguridad y salud en el trabajo}

La SST ha sido objeto de interés de la OIT y de la OMS desde que estos organismos se constituyeron en 1919 y 1948, respectivamente, y sus planteamientos se reforzaron con la creación del Comité Mixto OITOMS en la década de 1950. Desde entonces, han venido generando propuestas y directrices de seguridad y salud en el trabajo para su aplicación práctica en los países miembros. Desde el trabajo, los lineamientos surgen con la creación de la OIT, en 1919, como parte del Tratado de Versalles, y a partir de las ideas ya experimentadas en la Asociación Internacional para la Protección de los Trabajadores, fundada en Suiza en 1901. Hasta la fecha, la OIT es la única organización mundial de composición tripartita en sus órganos ejecutivos (29). Desde su Constitución, aborda la "protección del trabajador contra enfermedades, sean o no profesionales, y contra los accidentes de trabajo” (30), lo que reafirma con la Declaración de Filadelfia en 1944, al plantear el cuarto principio "todos los seres humanos tiene derecho a perseguir su bienestar [...]" (31). La OIT expresa sus principios, derechos y normas desde los convenios fundamentales, de 
gobernanza y técnicos, las recomendaciones, las guías y los protocolos (32. Los convenios son tratados vinculantes que pueden ser ratificados por los países miembros, y Colombia lo ha hecho con 61 de ellos, 54 de los cuales están en vigor (33), entre los que sobresale, para el caso, el C 161 de 1985 - Convenio sobre los servicios de salud en el trabajo (34), que indica las disposiciones para el establecimiento de estos servicios para conservar un medio ambiente de trabajo seguro y sano. No ratificados por el país, pero no por ello no aplicados, también se encuentran, el Convenio 155 de 1981 y su Protocolo del 2002 que insta a definir una política nacional de SST que tenga por objeto prevenir los accidentes y las enfermedades laborales (12), y del Convenio 187 y su Recomendación 197, ambos del 2006, que constituyen el marco promocional para la SST, por el que todo miembro deberá promover la mejora continua mediante la elaboración de una política, constitución de su sistema, diagnóstico de su perfil y sus programas nacionales correspondientes (14).

En efecto, para el nuevo milenio y teniendo en cuenta que en ese momento el mundo vivía incremento del desempleo, aumento de migraciones de trabajadores no calificados, inestabilidad laboral, deterioro de las condiciones laborales, entre otros (35), la OIT lanza su propuesta de Decent Work (Trabajo Decente), cuya finalidad es dar oportunidades a las personas para que puedan conseguir un trabajo productivo en condiciones de libertad, equidad, seguridad y dignidad humana (36), y publica las Directrices relativas a los sistemas de gestión de la seguridad y salud en el trabajo ILO-OSH: 2001, como referente para establecer la política nacional y la promoción de los sistemas de gestión de seguridad y salud en el trabajo (37). En el 2003 y con el propósito de honrar la memoria de los trabajadores lesionados, enfermos y fallecidos, la OIT lanza la campaña 28 de abril para prevenir y reducir los desenlaces de salud por exposición a riesgos laborales (38). Ese mismo año, y a partir de las conclusiones de la 91 Conferencia Internacional del Trabajo, se contempla que "El trabajo decente debe ser trabajo seguro", y sobre esta reflexión se adopta en el 2004 la "Estrategia Global en Materia de Seguridad y Salud en el Trabajo SST" (23, que junto con la Declaración de Seúl, que en 2008 reconoce la seguridad y salud en el trabajo como un derecho fundamental (39), confirman el rol de la OIT en la promoción de la SST y exhortan a implementar el Marco Promocional, para caracterizar las políticas, los planes y los perfiles nacionales como herramienta de seguimiento a los sistemas nacionales de seguridad y salud en el trabajo (17). Ad laterum la consideración que Decent Work hace parte integral de la agenda 2015-2030 de los Objetivos de Desarrollo Sostenible de Naciones Unidas (40), haciéndose absolutamente visible para las agendas públicas nacionales que no solo le apuestan a la oportunidad de tener trabajo remunerado y con derechos, sino que refuerzan la seguridad en el trabajo y los entornos laborales saludables. La última consideración, a la fecha, por parte de la OIT es la campaña del Día Mundial de la Seguridad y la Salud en el Trabajo para el 2017, que se centra en la necesidad de los países de mejorar su capacidad para recopilar y utilizar datos fiables sobre seguridad y salud en el trabajo (41).

Ahora bien, desde la salud, las directrices nacen consustanciales a la Oficina Sanitaria Panamericana, hoy Organización Panamericana de la Salud (OPS), que se constituye en 1902 como respuesta de los países de las Américas ante las epidemias del siglo XX (42). La OPS desde sus comienzos cuenta con doble rol sanitario, uno como la agencia especializada del Sistema Interamericano y el otro como la oficina regional para las Américas de la OMS (43). La OMS se origina en el seno de la Conferencia Sanitaria Internacional celebrada en Nueva York a mediados de 1946 y su constitución entra en vigor dos años más tarde. Esta organización, con independencia de las modificaciones adoptadas, ha mantenido sus “principios básicos para la felicidad, las relaciones armoniosas y la seguridad de todos los pueblos" (44).

La OMS y la OPS, acompaando a la OIT y de manera paralela con las propuestas de intervención sanitaria, han respaldado las acciones de salud laboral desde la política mundial plasmada en 1978 durante la primera Conferencia Internacional sobre Atención Primaria en Salud, conocida como la Declaración de Alma Ata, en la que se plantea llevar el bienestar a las personas, no solo al lugar donde viven sino hasta el lugar donde trabajan (45). De allí surge el concepto de cobertura universal, que promueve "Salud para Todos en el año 2000" y que se aplica a la SST como la Estrategia Mundial de la Salud Ocupacional para Todos: El camino hacia la salud en el trabajo (46). que refuerza "el lugar de trabajo como un entorno 
prioritario para la promoción de la salud en el siglo XXI puesto que la salud en el trabajo y los ambientes de trabajo saludables son los bienes más preciados de las personas" (47).

Adicionalmente, en consideración a que la Constitución de la OMS, artículo 2.i., requiere "promover [...] la mejora de las condiciones de trabajo" (44) y en concordancia con los resultados del ejercicio Estrategia de Promoción de la Salud en los Lugares de Trabajo de América Latina y el Caribe (47), el Plan Regional de Salud de los Trabajadores (48) y las recomendaciones de la Cumbre Mundial sobre el Desarrollo Sostenible, las cuales refuerzan la labor de la OMS en el ámbito de la SST (49), se genera una cascada de propuestas para la presente década que promueven el fortalecimiento de la voluntad política de intervenir los lugares de trabajo y la gestión de riesgos que prevengan accidentes y enfermedades profesionales, como lo sugieren la Carta de Bangkok, Promoción de la salud en el mundo globalizado, resultante de la VI Conferencia Internacional sobre la Promoción de la Salud (50), la Declaración de Stresa y su marco para la acción concertada de todas las partes, con el fin de proteger y promover la salud de los trabajadores (51), y la propuesta de la 60 Asamblea Mundial de la Salud con el Plan Global de Acciones para la Salud de los Trabajadores (GPA) 2008-2017, que establece: "Diseñar e implementar políticas para normar la salud de los trabajadores, proteger y promover la salud en el lugar de trabajo, promover la implementación y el acceso a los servicios de salud ocupacional, proporcionar y comunicar las evidencias de acción y práctica e incorporar la salud de los trabajadores en otras políticas" (52), el cual se extiende con el modelo Entornos Laborales Saludables, que a su vez se constituye en la guía para lograr salud en el trabajo a expensas de espacios de trabajo libres de riesgos (26).

En la actualidad, en consonancia con el Plan de acción sobre la salud en todas las políticas, las nuevas metas de desarrollo sostenible de las Naciones Unidas, el Plan Estratégico de la OPS 2014-2019, entre otros, la OPS y la OMS postulan el Plan de acción sobre la salud de los trabajadores 2015-2025, dirigido a proteger la vida, promover la salud y el bienestar de los trabajadores, con énfasis en quienes estén expuestos a condiciones de trabajo peligrosas (53).

De otra parte y no menos importante, desde el 2012 la OIT ha desarrollado una estrategia bidimensional de extensión de la protección que permita universalizar y mejorar las prestaciones de la seguridad social y en coordinación con la OPS avanzan en la propuesta de políticas públicas que refuerzan la protección social en la región (54). En la figura 1 se esquematiza la evolución temporal de las iniciativas y estrategias globales en materia de seguridad y salud en el trabajo.

\section{ORGANIZACIÓN INTERNACIONAL DEL TRABAJO}

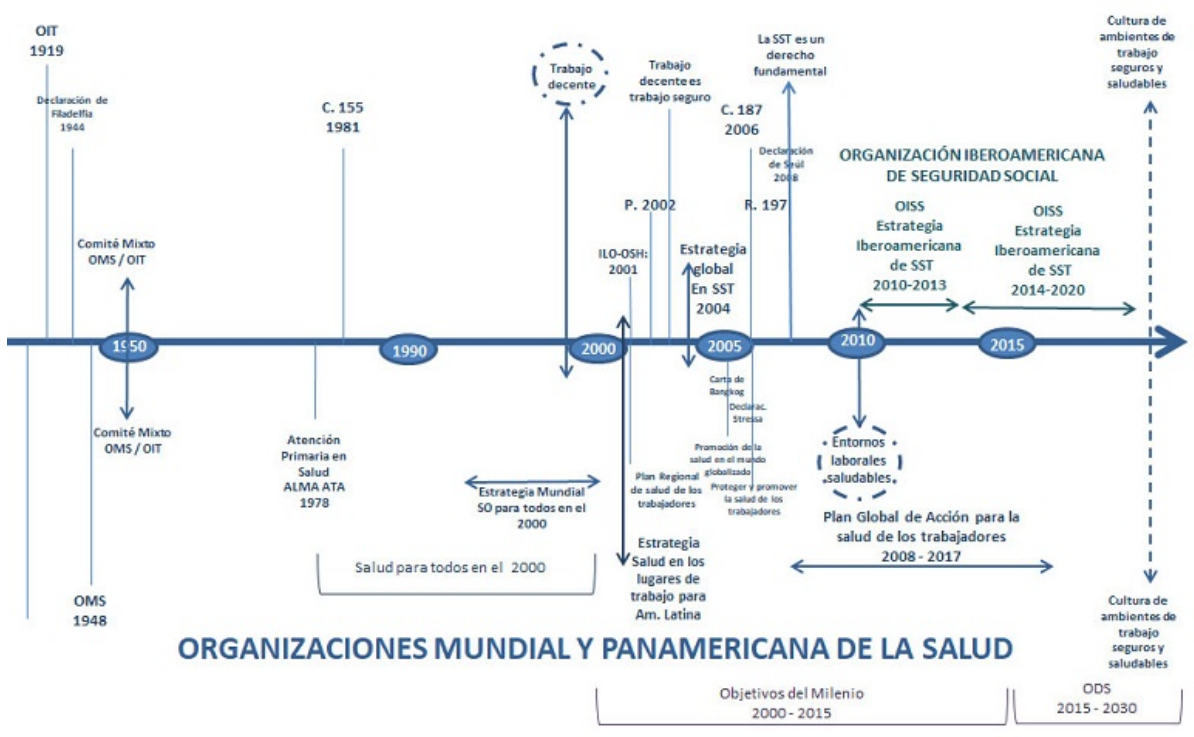

FIGURA 1

Iniciativas y estrategias globales en materia de seguridad y salud en el trabajo Fuente: Elaboración propia. 


\section{La evolución del mundo del trabajo y las iniciativas regionales}

En el siglo XXI las formas de trabajo se han diversificado, la población mundial está envejeciendo y la sociedad se enfrenta a grandes retos relacionados con la pobreza, las migraciones, la expectativa de vida, el empleo, nuevos riesgos ocupacionales y ambientales, entre otros. Todo esto hace que en Europa se impongan marcos reguladores para mejorar la calidad del trabajo, lo que se busca instando a los actores a poner en práctica el vasto acervo de instrumentos de seguridad y salud en el trabajo generados por la OIT - adoptados por los países miembro y expresados como legislación nacional que debe impulsarse y la política de "trabajo decente" en proceso de implementación-y la OMS - consolidando la cultura de prevención de los riesgos laborales y el planteamiento de bienestar en el trabajo para garantizar entornos saludables-, lo que da como resultado la Estrategia Comunitaria SST 2002-2006 (55), seguida de la II Estrategia Europea sobre Seguridad y Salud en el Trabajo 2007-2013, y ahora la III Estrategia Europea sobre Seguridad y Salud en el Trabajo 2014-2020. La Unión Europea ha visto una reducción en el número de accidentes de trabajo y enfermedades laborales. Pese a esto, continúa sugiriendo la integración de la SST a las políticas comunitarias por sus repercusiones en el bienestar de los trabajadores, la productividad empresarial y el crecimiento económico y en consideración al tamaño de las empresas, los riesgos emergentes y los cambios demográficos que se avecinan (56).

Latinoamérica hace lo propio desde la Comunidad Andina de Naciones (CAN), que en 1999, mediante la Declaración de Cartagena de Indias, empieza a proyectar un direccionamiento para impulsar integración política, económica, cultural y social (57) para procurar el mejoramiento del nivel de vida de sus habitantes, intrínsecamente relacionado con la obtención de un trabajo decente, que culmina cuatro años después con la Decisión 584 del 2004, la cual adopta el Instrumento Andino de Seguridad y Salud en el Trabajo, que refuerza la puesta en práctica o revisión de una política de prevención de riesgos laborales (58). Lo anterior, sumado a los compromisos internacionales con los derechos humanos a la seguridad social, al trabajo y a la salud (59), conduce a la Organización Iberoamericana de Seguridad Social (OISS) a que presente, en el 2006, la Declaración Iberoamericana sobre Seguridad y Salud en el Trabajo y que al año siguiente exprese el Acuerdo de Voluntades, animando a promover la cultura de prevención de riesgos laborales y la gestión del trabajo decente (60). La OISS continúa haciendo lo propio, en consideración a que los derechos a la vida, al trabajo y a la salud son consustanciales a la prevención de accidentes y enfermedades laborales y se adhiere al precepto de que "las políticas públicas en seguridad y salud en el trabajo se constituyen en el frente esencial para articular respuestas que coadyuven al bienestar de las personas que laboran", emitiendo la Estrategia Iberoamericana de Seguridad y Salud en el Trabajo 2010-2013, que se acompañó de la creación del Observatorio Iberoamericano de Riesgos Profesionales, de la puesta en marcha del Sistema Armonizado de Indicadores de Siniestralidad (SIARIN) y de la invitación a gobiernos de la región para establecer actuaciones prioritarias sobre sus sistemas, políticas y programas nacionales de SST (61). Este, junto con el objetivo 8 de trabajo decente y crecimiento económico de los Objetivos de Desarrollo del Milenio (40), fueron los impulsores de la II Estrategia Iberoamericana de Seguridad y Salud en el Trabajo 2015-2020, dirigida a la consecución de instrumentos técnicos y fórmulas concretas para progresar en el protagonismo de los agentes sociales y en el dialogo social; en la promoción de las políticas y programas nacionales de SST; fomentar la educación, la cultura preventiva, procesos de investigación, desarrollo e innovación sobre temas de SST, con el fin de promover la reducción de los riesgos laborales, accidentes, enfermedades y muertes en los lugares de trabajo y avanzar sustancialmente en la mejora del trabajo decente para todos los trabajadores de la región $(62)$. 


\section{Material y Métodos}

Este artículo es una revisión narrativa de tipo cualitativo y su realización se hizo a partir de la búsqueda, selección y análisis de la literatura especializada en políticas públicas y de seguridad y salud en el trabajo y los aportes de estas a Colombia. Se realizaron consultas en las bases de datos Academic Search Premier, SciELO, Directory of Open Acces Journal (DOAJ), Fuente Académica Premier, Science Direct, Business Source Complete y JSTOR. Se usaron los términos Decs política pública, salud laboral y programas nacionales de salud, y los $\mathrm{MeSH}$ public policy, occupational health y National health programs; adicionalmente, se hizo otra pesquisa que incluyó Colombia. Los resultados sin limitaciones y en español arrojaron 24 artículos, de los cuales uno correspondía a la temática requerida y presentaba desde una revisión de la literatura, que en Latinoamérica y en especial en Colombia no se tienen estudios al respecto (63); en inglés se obtuvieron 390 artículos, solo uno relacionado con la indagación de esta revisión, y se refiere la necesidad de fortalecer la capacidad de investigación, capacitación y formulación de políticas de salud ambiental y ocupacional en el país (64). Posteriormente, y a partir de la experiencia de las autoras, se exploró literatura gris en materia de seguridad y salud en el trabajo, como resultado de lo cual se encontraron, básicamente, informes y documentos de organismos internacionales, reportes institucionales, políticas nacionales de Colombia afines a la temática, y también se revisó la legislación correspondiente de salud ocupacional y riesgos laborales. La información se organizó desde el punto de vista teórico-conceptual y se llegó a un consenso entre las autoras para mostrar los resultados, describiendo los lineamientos mundiales, las estrategias de Iberoamérica en política pública y los programas nacionales de Colombia en materia de SST.

\section{Resultados}

\section{La política pública y los planes nacionales de salud ocupacional en Colombia}

El primer acercamiento legislativo a la salud de los trabajadores se encuentra en el artículo 44 de la Constitución Política de la República de Colombia de 1886, hoy en día derogada, en donde se leía "toda persona podrá abrazar un oficio u ocupación [...] y las autoridades inspeccionarán las industrias y procesiones en lo relativo a la moralidad, la seguridad y la salubridad" (65). Sin embargo, es con la Ley 57 de 1915, Ley Uribe-Uribe, cuando aparecen los primeros esfuerzos legislativos a expensas de la reparación de los infortunios por accidentes de trabajo (66). En los años siguientes, Colombia ratifica convenios técnicos de la OIT (67) relacionados con horas de trabajo, trabajo nocturno, indemnizaciones por accidentes de trabajo, enfermedades profesionales y otros (33), que soportan las bases de la Ley 6 de 1945-Ley General del Trabajo (68), la cual establece disposiciones para asociaciones profesionales, conflictos colectivos, convenciones de trabajo y facilita la organización de la Caja de Previsión Social, para dar cobertura a los empleados oficiales, y la creación del Seguro Social, Ley 90 de 1946, para los trabajadores del sector privado. Ambas entidades cubrían salud, pensiones y eventos relacionados con las labores desempeñadas, prestaciones patronales. Mediante la Ley 77 de 1948 se crea la Oficina de Medicina e Higiene Industrial, cuyo funcionamiento se reglamenta con el Decreto 3767 de 1949 (69).

La década de 1950 empieza con la expedición del Código Sustantivo del Trabajo, que estableció con rango legal las medidas de higiene y seguridad en el trabajo, tipos de contratación, jornadas de trabajo y prestaciones asistenciales y económicas por accidentes y enfermedades laborales (70). El componente seguridad social y riesgos del trabajo se empieza a desarrollar en las siguientes dos décadas, que el país aprovecha, entre otros aspectos, para ratificar otros convenios OIT afines a SST, como los de trabajo forzoso, igualdad de remuneración, libertad sindical, negociación colectiva, descanso semanal, vacaciones pagas y el de discriminación por empleo y ocupación (71). Mientras tanto, Colombia y todos los demás países de la región andina suscriben el Pacto Internacional de Derechos Económicos, Sociales y Culturales, que entra en vigor en 1976, el cual reconoce que el derecho a la salud en el trabajo es un derecho humano fundamental, por lo que se reconoce a las personas el disfrute del más alto nivel posible de salud física y mental (72). 
En 1979, Colombia entra oficialmente al marco legislativo de la seguridad y salud en el trabajo, desde tres campos de acción, vigentes hoy en día, a saber: regulaciones de la salud de los trabajadores, sobre los ambientes de trabajo y para inspección vigilancia y control, empezando con la Ley 9 de 1979 Código Sanitario Nacional, que establece "Preservar, conservar y mejorar la salud de los individuos en sus ocupaciones" (73), reglamentada en la Resolución 2400 de 1979 con disposiciones sobre higiene y seguridad en los establecimientos de trabajo (74), seguida de la Política de Trabajo y Seguridad Social de 1980, ofrecida en el Plan Nacional de Desarrollo,en ese entonces Plan de Integración Nacional 1979-1983, pero que es más una política laboral dirigida a aumentar la productividad y el empleo (75).

Con el fin de armonizar las acciones y competencias de las entidades públicas y privadas en lo relacionado con seguridad y salud en el trabajo, en 1983 se constituye el Comité Nacional de Salud Ocupacional, cuya primera tarea es elaborar el Plan Nacional de Salud Ocupacional 1984, con el objeto de "implementar una política de salud para los trabajadores en el territorio nacional" (76), requerido como un plan nacional unificado en el campo de la prevención de los accidentes y las enfermedades relacionadas con el trabajo y del mejoramiento de las condiciones de trabajo por el Decreto 614 del mismo año, el cual señalaba las bases para la organización de la salud ocupacional en el país (77). Precisamente, como parte del desarrollo de este plan, se expide la reglamentación del Programa de Salud Ocupacional (78), cuyas actividades están dirigidas a prevenir los accidentes y las enfermedades, y el cual se complementa con la institucionalización de los comités paritarios (empresa-trabajadores) con el propósito de promover y vigilar las intervenciones de salud ocupacional en el interior de la empresa (79). También se adopta la primera tabla de enfermedades profesionales del país (80).

El Plan Nacional de Salud Ocupacional 1990-1995, propuesto en el marco del Primer Congreso Nacional de Entidades Gubernamentales de Salud Ocupacional y elaborado en el seno de los ministerios de Trabajo y Seguridad Social y de Salud y por el Instituto de Seguros Sociales, tiene entre sus propósitos "reducir la ocurrencia de accidentes de trabajo y la aparición de enfermedades profesionales" (76) y sus intervenciones van dirigidas a la legislación, investigación, educación, vigilancia y control y sistema de información en salud ocupacional (81). En los noventa, Colombia está pasando de Estado de Bienestar a Estado Social de Derecho, que se configura con la Constitución Política de 1991, que hoy garantiza a todos los habitantes el derecho a la seguridad social, el acceso a los servicios de promoción, protección y recuperación de la salud y contempla el trabajo como un derecho en condiciones dignas y justas (73). Se crea el Sistema de Seguridad Social Integral, desde la Ley 100 de 1993 (70), cuya operación gira en torno a pensiones, salud y riesgos profesionales, cuya organización y administración están dadas por el Decreto 1295 de 1994 (82) y que está conformado por: las administradoras de riesgos laborales (ARL) públicas y privadas, los empleadores y los trabajadores, las entidades encargadas del control y la vigilancia del sistema, como el Ministerio de Salud y Protección Social, el Ministerio del Trabajo, la Superintendencia Nacional de Salud, la Superintendencia Financiera, el Consejo Nacional de Riesgos Laborales, el Comité Nacional de Salud Ocupacional y los comités regionales y locales de salud ocupacional (83). Y es precisamente mientras se da la reglamentación del SGRL que se produce la brecha de ocho años en la continuidad de los planes nacionales.

El que Colombia no se haya adherido al Convenio sobre seguridad y salud de los trabajadores de OIT, el C-155 de 1981 (33), no es obstáculo para presentar en el 2001 su directriz nacional de SST, emanada de la Dirección de Salud Ocupacional y Riesgos Profesionales del Ministerio de la Protección Social, promulgando "La política pública para la protección en el mundo del trabajo", que tiene por objeto "convocar a la sociedad en su conjunto, para que de manera permanente, a través de procesos de participación, construcción colectiva, articulación, alianzas estratégicas y sinergia de recursos, se logre para la población económicamente activa del país mejores condiciones en sus lugares de trabajo como garantía de un derecho constitucional, calidad de vida y como aporte al proceso de paz" (84). El 
alcance de esta es a través de cinco ejes, a saber: construcción de una cultura de seguridad social y prevención de los riesgos laborales; fortalecimiento institucional; intervención sobre las poblaciones especiales; desarrollo técnico-científico y sistemas de gestión (85), que a su vez se constituyen en referentes para los planes nacionales subsecuentes. Al año siguiente se expide la Ley 776 del 2002, que dicta normas adicionales y formaliza las prestaciones del SGRL (86); además, se publica el primer Informe de Enfermedad Profesional en Colombia 2000-2002 (87).

Conocedor del subregistro de los accidentes y subdiagnóstico de las enfermedades, el país presenta el Plan Nacional de Salud Ocupacional 2003-2007, cuyo primer objetivo es "la prevención de los riesgos profesionales" (88). Sin embargo, consciente de lo novedoso del tema, dirige sus acciones hacia el conocimiento de los conceptos de ATEL y establece que se adopten los formatos de notificación e informe de accidente de trabajo y enfermedad profesional: FURAT y FUREP (89); define las actividades de alto riesgo; elabora las guías de atención integral en salud ocupacional basadas en la evidencia (Gatiso) para las diez principales causas de morbilidad profesional $(90,91)$. Y a partir del Informe de Enfermedad Laboral en Colombia 2001-2002 refuerza la práctica de evaluaciones médicas de ingreso, periódicas y de retiro, los diagnósticos de condiciones de salud (92), archivo de historias clínicas (93). También expide reglamentación que obliga a los empleadores a investigar todos los casi-accidentes y los accidentes de trabajo (94) y publica el Diagnóstico actual y prospectivo de la salud ocupacional con enfoque de entornos (95), y en el 2006 realiza la Primera Encuesta Nacional de Condiciones de Salud y Trabajo en el Sistema General de Riesgos Profesionales (96), la cual se constituye en la línea de base para el plan que le siguió.

Así mismo, en el 2006 Colombia participa del ejercicio de la OIT para realizar los perfiles nacionales de la región andina, reportando, entre otros, ratificación de nuevo convenios relacionados con SST, 25\% de cobertura de la legislación y $31 \%$ de cobertura del aseguramiento en riesgos laborales, con estimados más de 2,5 millones de accidentes de trabajo (97).

El PNSO 2008-2012 da continuidad a los objetivos el plan anterior, resaltando la necesidad de fortalecer "la promoción de la salud de los trabajadores y la prevención de los riesgos para disminuir la frecuencia y severidad de los accidentes de trabajo y enfermedades profesionales" (98). Se desarrolla en paralelo con el Plan Nacional de Salud Pública 2007-2010, que ordena la articulación de las administradoras de riesgos laborales (ARL) con las entidades territoriales para mejorar la SST, que a su vez está descrita como una de las 10 prioridades de salud pública del país y a la que le aplica "Implementar y evaluar la estrategia de entornos saludables en espacios educativos, de vivienda y espacios laborales" y, puntualmente, "Promover la implementación y evaluación de la efectividad de la estrategia de entornos saludables en espacios laborales" (99). Las intervenciones se dan a través de la implementación de reglamentos técnicos, programas de vigilancia epidemiológica ocupacional y estrategias de prevención de acuerdo con la morbimortalidad; se actualiza la tabla de enfermedades profesionales (100), se reglamenta el trabajo en alturas (101); se emite el Reglamento de Salud Ocupacional en procesos de energía; se adopta el Reglamento de higiene y seguridad con crisolito; se establece el Sistema de Garantía de Calidad en Salud Ocupacional y Riesgos Profesionales (102) y se realiza la Segunda Encuesta Nacional de Condiciones de Salud y Trabajo en el Sistema General de Riesgos Laborales (103).

En el país, la segunda década del siglo XXI plantea un impulso a las intervenciones de salud, de salud pública y de salud ocupacional que se concretan con reformas a la seguridad social, incluyendo riesgos profesionales, modificado con la Ley 1562 del 2012 (104), que cambia su nombre a Sistema General de Riesgos Laborales (SGRL), el cual adicionalmente determina que "de ahora en adelante salud ocupacional se entenderá como seguridad y salud en el trabajo y el programa de salud ocupacional, en lo sucesivo, será un sistema de gestión de seguridad y salud en el trabajo". En este contexto y con el enfoque de gestión sistemática de la SST, por medio de acciones dirigidas al fomento de estilos de trabajo y de vida saludables, entornos laborales seguros y prevención de los accidentes y las enfermedades en los trabajadores, surge el Plan Nacional de Seguridad y Salud en el Trabajo 2013-2021, el cual se proyectó "en el marco del cumplimiento de los compromisos asumidos por el gobierno nacional para dar continuidad a los ejes de la 
Política Pública para la Protección de la Salud en el Mundo del Trabajo", la articulación con el Plan Decenal de Salud Pública 2012-2021 en la dimensión Salud y Ámbito Laboral e indicando como referentes nacionales a la reglamentación técnica y los resultados de la II Encuesta Nacional de Condiciones de Salud y Trabajo, con datos de 2013, y como internacionales las Estrategias Iberoamericanas de Seguridad y Salud en el Trabajo 2010-2014 y 2015-2020, el Diálogo Social y Trabajo Decente (105).

El PNSST se estructura con un fin último: "el fomento de una cultura preventiva en todos los niveles, que se refleje en el bienestar y calidad de vida de los trabajadores", cuyo impacto se evalúa desde "el fomento a la salud, la calidad de vida y de trabajo" y "la reducción de la siniestralidad" (106). Aunque el PNSST 2013-2021 se encuentra en desarrollo, ya se ha presentado el primer reporte de su seguimiento (85), elemento que retoma la importancia de evaluar la política pública nacional en materia de seguridad y salud en el trabajo, en términos de progreso, desempeño e impacto sobre la prevención de accidentes de trabajo y enfermedades laborales.

En este momento, es oportuno precisar que los trabajadores afiliados al SGRL son aproximadamente diez millones de personas y tienen cubiertas sus prestaciones asistenciales y económicas; sin embargo, en Colombia se calcula que otro tanto (107) están trabajando sin protección, lo que los hace población vulnerable y por ende objeto de atención del Plan Decenal de Salud Pública de Colombia 2012-2021, en la dimensión de salud ambiental, componentes hábitat saludable y protección de la salud de los trabajadores, con base en las estrategias de entornos laborales saludables, promoción de la salud de los trabajadores informales, mitigación de los riesgos laborales y prevención de las enfermedades y accidentes de trabajo (108).

Como complemento a las acciones de los programas de SST, el país también se encuentra implementando el Plan Nacional de Desarrollo 2014-2018 con la visión prevista por los Objetivos de Desarrollo Sostenible. En este plan, la SST está respaldada por el impulso del Diálogo Social y la concertación laboral, la prevalencia de los derechos fundamentales del trabajo y la sostenibilidad de las empresas y la adopción de la Política Nacional de Trabajo Decente, para promover la generación de empleo, la formalización laboral y la protección de los trabajadores (109).

En el 2015 se establece la celebración del Día del Trabajo Decente, teniendo en cuenta como ejes temáticos: la promoción del diálogo social, la creación de trabajo, el cumplimiento de los derechos de los trabajadores y la extensión de la protección social que contempla, entre otros, velar por que las personas disfruten de condiciones de trabajo seguras y la productividad empresarial (110). En la figura 2 se presenta la síntesis de la evolución de las iniciativas nacionales en seguridad y salud en el trabajo para Colombia.

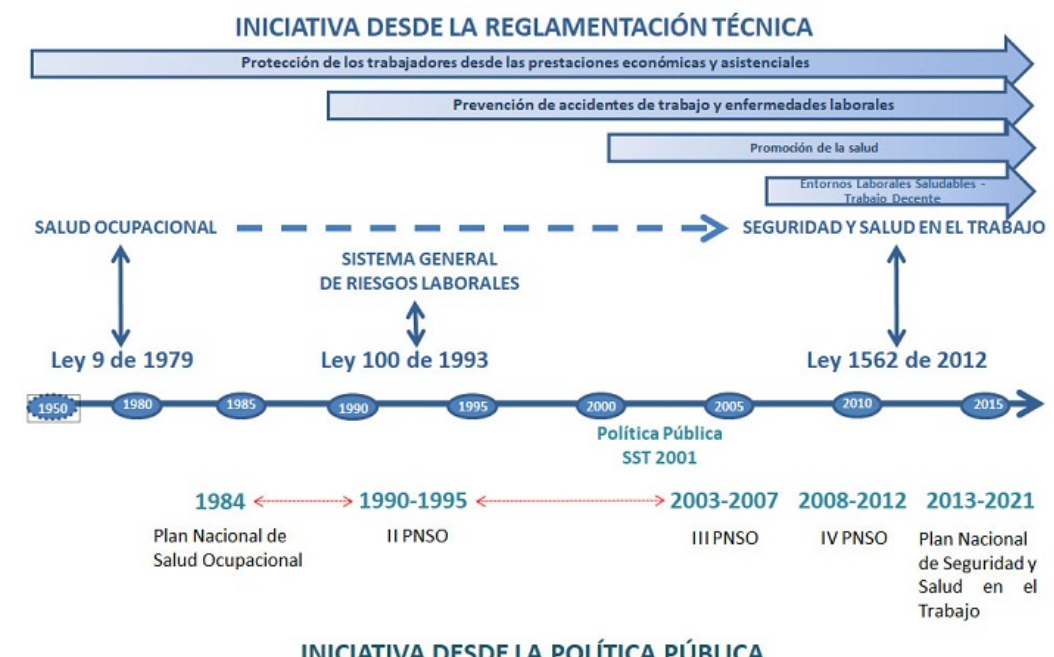

FIGURA 2

Colombia. Iniciativas nacionales en seguridad y salud en el trabajo Fuente: Elaboración propia. 


\section{Discusión}

Las acciones conjuntas entre la OIT y la OMS han instado a los gobiernos a seguir el marco promocional de la SST para gestionar los riesgos y prevenir los ATEL, a partir de una propuesta metodológica de política, sistema, perfil y programas nacionales. La OISS ha hecho acopio de este modelo a través de las estrategias iberoamericanas y ha apoyado a Colombia en la implementación de la SST con enfoque de la cultura de prevención en entornos saludables. El país en el 2001 presenta su Política pública para la protección en el mundo del trabajo, de la cual solo se reconocen: su objeto, en los planes nacionales 2003-2007 y 2008-2012, y sus ejes en el Plan Nacional SST 2013-2021. A partir de estos, se puede evidenciar que:

- Aunque tiene por objeto "convocar a la sociedad en su conjunto, para que, de manera permanente, a través de procesos de participación, construcción colectiva, articulación, alianzas estratégicas y sinergia de recursos, se logre para la población económicamente activa del país, mejores condiciones en sus lugares de trabajo como garantía de un derecho constitucional, calidad de vida y como aporte al proceso de paz", no se encontró una declaración escrita formalmente, ni respaldada por acto administrativo, desconociéndose el ejercicio de construcción tripartita. Sin embargo, es retadora al plantearse como objeto a la población económicamente activa, como bien lo sugiere la OMS con Salud para Todos en el año 2000, porque tres quinquenios después, la cobertura del SGRL es de un 50\% de dicha población, básicamente trabajadores formales.

- De acuerdo con el marco promocional, las políticas nacionales específicas de SST expresan claramente el compromiso de las autoridades públicas, los propósitos del mandato, sus principios orientadores y las esferas de acción. Los ejes de la política colombiana se pueden entender como i) compromiso: el fortalecimiento institucional; ii) propósitos: la cultura de seguridad social y la prevención de los riesgos laborales, ATEL; iii) orientadores: el desarrollo técnico-científico; y iv) esferas de acción: los sistemas de gestión y la intervención sobre las poblaciones especiales. Claro está que si sobre la letra está el espíritu de la ley, en este caso sin documentación formal se pone de manifiesto que a la fecha se desconoce la intención de quienes la construyeron y en este punto los autores solo hacemos una interpretación a posteriori.

Desde hace veinticinco años, las acciones de seguridad y salud en el trabajo en Colombia se han concebido a partir del Sistema General de Riesgos Laborales, que "es el conjunto de entidades públicas y privadas, normas y procedimientos destinados a destinados a prevenir, proteger y atender a los trabajadores de los efectos de las enfermedades y los accidentes que puedan ocurrirles con ocasión o como consecuencia del trabajo que desarrollan" y que hace parte estructural del Sistema de Seguridad Social Integral. El país ha desarrollado toda una experiencia con el SGRL, actualmente a cargo del Ministerio del Trabajo, y cuenta con el Consejo Nacional de Riesgos Laborales, el Comité Nacional de Seguridad y Salud en el Trabajo y el Fondo de Riesgos Laborales, que comparten acciones con las agremiaciones empresariales y los representantes de los trabajadores. No es objeto de discusión en este artículo, pero con respecto a los elementos requeridos por el marco promocional de la SST, en la práctica no cuenta con subsistema de información propio y es conocido el peso de estos datos en la toma de decisiones de la política pública.

Los perfiles nacionales se consideran la herramienta fundamental para realizar los programas, pues además de caracterizar la situación del país en materia de SST, identifican puntos fuertes y débiles que sugieren las acciones que es preciso priorizar. Colombia tiene un perfil estandarizado a requerimientos OIT, realizado en el 2006 y que aplicó junto con los resultados de la I Encuesta de Condiciones de Salud y Trabajo, para el establecimiento del Plan Nacional de Salud Ocupacional 2008-2012. El país ha venido reforzando su diagnóstico de situación actual en SST con la II Encuesta de Condiciones SST en el SGRL, antesala del Plan Nacional de SST 2013-2021. No obstante, se hace necesario elaborar otro perfil nacional que, como bien lo indican las estrategias mundiales, es un mecanismo de gestión que al aplicarse de manera consecutiva permite evaluar progresos, incluso resultados de los programas e impacto de la política. 
Desde la década de los ochenta, el país ha requerido planes de acción para prevenir los ATEL, como se advierte desde el Plan Nacional de Salud Ocupacional de 1984, pasando por los planes nacionales 1990-1995, 2003-2007 y 2008-2012, hasta la propuesta vigente de Plan Nacional de Seguridad y Salud en el Trabajo 2013-2021. Su objeto, autores y antecedentes se muestran tabla 1, en la que se observa cómo han venido madurando los programas nacionales en la medida que se han ido adhiriendo a los lineamientos globales y ajustando a las estrategias regionales.

- En el caso del objeto de los programas nacionales, la evolución va desde implementar la política, pasando por la prevención de ATEL, fomento del autocuidado, promoción de la SST hasta creación de una cultura de prevención con reducción de la siniestralidad laboral.

- Los autores empiezan siendo los entes de dirección y coordinación del sistema. Una vez instituido el SGRL, se ve el enfoque tripartito cuando a los entes gubernamentales se unen los representantes de los trabajadores y los empleadores, incluso las aseguradoras, y los planes de esta década empiezan a involucrar a los institutos de salud, a la academia y a centros de investigación.

- En los antecedentes nacionales, la política pública es protagonista en los planes nacionales de este milenio, junto con los planes nacionales de desarrollo y los planes de salud, a los que se unen el perfil país SST del 2006 y las encuestas nacionales de condiciones de SST 2007 y 2013.

- Los referentes internacionales solo son explícitos en el Plan Nacional SST, en curso, y entre estos la Decisión 584 de la CAN, las Estrategias Iberoamericanas de SST, el Diálogo Social y el Trabajo Decente.

TABLA 1

Características de los planes nacionales de SST

\begin{tabular}{|c|c|c|c|c|c|}
\hline Caracteristicas & 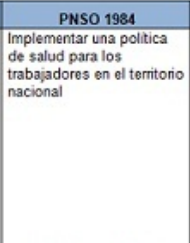 & 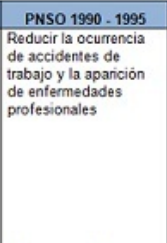 & 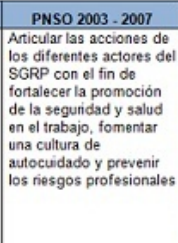 & 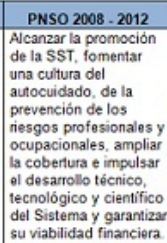 & 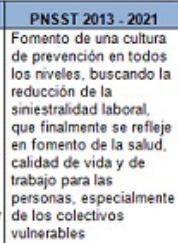 \\
\hline Autores & $\begin{array}{l}\text { Comité Nacional de Salud } \\
\text { Ocupacional }\end{array}$ & 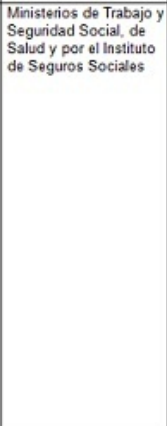 & $\begin{array}{l}\text { Ministerio de la } \\
\text { Protección Social } \\
\text { Con Reperesentantes del } \\
\text { Gobiemo Nacional, de } \\
\text { los trabajajadores, } \\
\text { empleadores } \\
\text { aseguradores. }\end{array}$ & 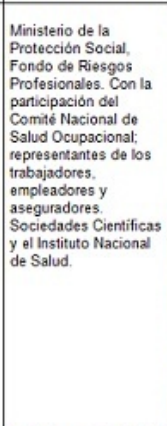 & 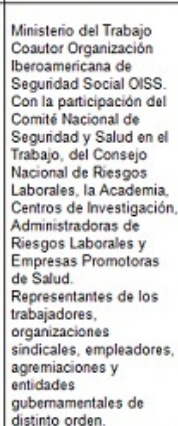 \\
\hline $\begin{array}{l}\text { Fecha de } \\
\text { publicación }\end{array}$ & & & Noviembre de 2004 & 2009 (Segunda Edción) & 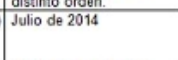 \\
\hline $\begin{array}{l}\text { Reglamentación que } \\
\text { lo respalda }\end{array}$ & & & & & 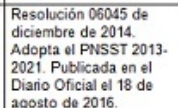 \\
\hline $\begin{array}{l}\text { Referentes } \\
\text { internacionsles }\end{array}$ & & & & & 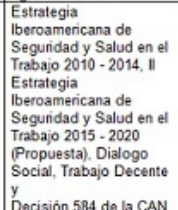 \\
\hline $\begin{array}{l}\text { Referentes } \\
\text { nacionales }\end{array}$ & & & 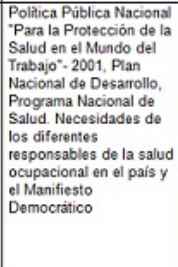 & 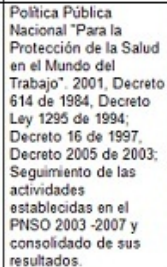 & 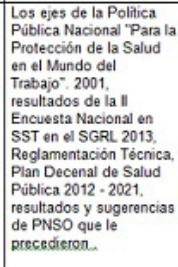 \\
\hline
\end{tabular}

Fuente: elaboración propia. 
Los planes nacionales de SST del nuevo milenio se han plasmado con líneas de acción e indicadores, descritos en la tabla 2, dirigidos primordialmente a fortalecer las instituciones para controlar la morbimortalidad laboral.

- Los planes nacionales de salud ocupacional 2003-2007 y 2008-2012 hacen parte de una misma línea gubernamental y por ende el segundo es continuidad del primero en el enfoque de herramienta de gestión para el desarrollo del SGRL, lo cual se configura con base en el fortalecimiento de las instituciones, la viabilidad financiera del sistema, el aumento de la cobertura, la optimización del reconocimiento de prestaciones, con impulso al desarrollo técnico, tecnológico y científico para la promoción de la SST. La disminución de los ATEL la deja en manos del sector empresarial, con el acompañamiento de las aseguradoras de riesgos laborales a través de sistemas de gestión de seguridad y salud en el trabajo (SG-SST). No tiene una referencia clara de los resultados, más allá del cumplimiento de actividades con sus respectivos indicadores.

- El Plan Nacional de Seguridad y Salud en el Trabajo 2013-2021 estructura sus líneas de acuerdo con los ejes de la política pública y las expresa como: fortalecimiento institucional, promoción de la SST y prevención de ATEL, optimización del reconocimiento de prestaciones y fomento de la transversalidad de la SST en el conjunto de las políticas públicas. Cada línea contiene sus objetivos generales con metas e indicadores y objetivos específicos con actividades, responsables y logros, lo que facilita el seguimiento de resultados, pero sobre todo la medición del impacto del plan nacional, como este mismo lo expresa, con la reducción de la siniestralidad laboral, el fomento de la salud y la calidad de vida y de trabajo para las personas, especialmente colectivos más vulnerables.

A partir del hecho de que el PNSST es coherente con la estrategia: política, sistema, perfil, programas nacionales, y que esta a su vez es proceso cíclico, el derrotero ahora es el seguimiento de los resultados y la evaluación del impacto del PNSST que contemple pertinencia de las prioridades y objetivos, eficacia y eficiencia de las intervenciones para dar vía libre al mejoramiento continuo que garantizará la verdadera transformación de la seguridad y salud en el trabajo.

TABLA 2

Áreas, obietivos v resultados de los planes nacionales de SST

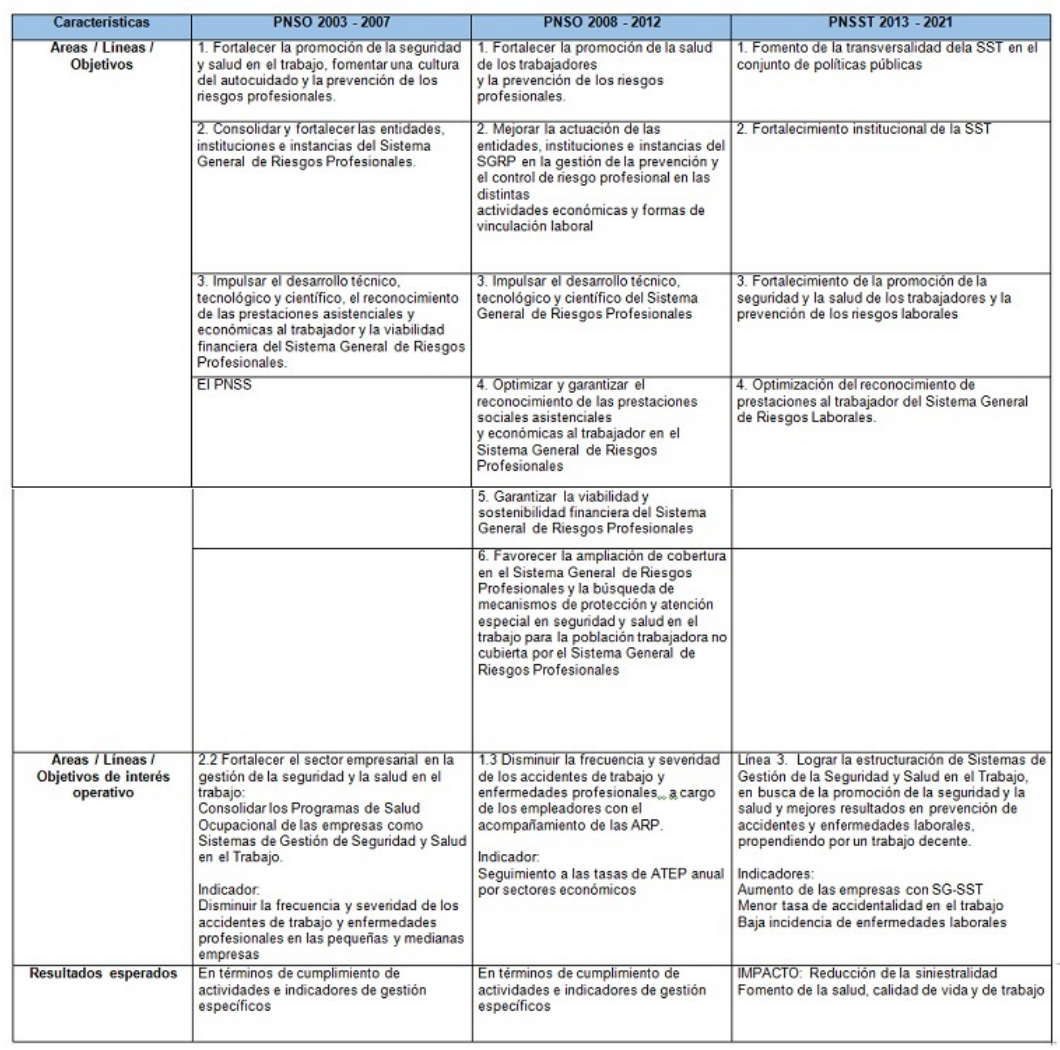

Fuente: elaboracion propia. 


\section{A modo de conclusión}

En la práctica, el marco promocional de la SST no obliga a la retificación de los convenios globales ni a la vinculación formal a las propuestas regionales, tampoco exige que la nación parta de ceros en SST, por el contrario, es un modelo flexible y dinámico que se ajusta al comportamiento y al nivel de desarrollo e infraestructura de cada país. Colombia ha intervenido la relación hombre-trabajo desde hace más de un siglo. En los últimos 50 años se ha venido ciñendo a los señalamientos supranacionales y a los compromisos globales, por lo que en la última década ha direccionado sus políticas con el enfoque de derechos, ha seguido a la OIT y a la OMS con sus postulados de cultura de prevención para entornos laborales saludables, y se está apoyando en la OISS para proyectar sus programas nacionales de SST. Sin embargo, aunque la política pública de SST está formulada, no es evidente su socialización a la ciudadanía, como tampoco que esté siendo examinada periódicamente y con rendición de cuentas por su ejecución. Definitivamente, esta es una tarea pendiente.

El SGRL está dirigido a prevenir, proteger y atender a los trabajadores de los efectos de las enfermedades y los accidentes que puedan ocurrirles con ocasión o como consecuencia del trabajo que desarrollan, y en este las acciones se comparten entre el Gobierno, las empresas y sus aseguradoras y los trabajadores. No obstante, el sistema se modificó y aún se encuentra en proceso de ajuste a los cambios. No cuenta con un subsistema de información armonizado, lo que restringe la calidad de los datos de ATEL para actualizar el perfil nacional y garantizar un diagnóstico en tiempo real de la situación actual de la siniestralidad laboral, lo cual limita la evaluación de pertinencia de las intervenciones.

Es necesario propiciar la concepción holística de la SST, de tal forma que las políticas públicas nacionales se desplieguen con estrategias que respondan al contexto país y a las necesidades específicas de trabajadores y empresas. Y para Colombia implica revisar la formulación de la Política pública para la protección en el mundo del trabajo, socializarla y, entonces sí, desplegarla formalmente en sus planes nacionales de SST y salud pública, construidos colectivamente, de tal forma que los haga prácticos, dinámicos, y no solo en términos de cobertura, sino de impacto de la política pública sobre la problemática de la siniestralidad en SST.

El caso colombiano, visto desde la política pública y los planes nacionales de seguridad y salud en el trabajo, presenta la situación del país durante los últimos quince años, la cual por cierto corresponde al marco estratégico mundial, pero solo permite hacer una descripción operativa del modelo. No obstante, considerar una evaluación de impacto de la política pública Mejores condiciones de Trabajo, a partir del comportamiento de los accidentes de trabajo y las enfermedades laborales, permitiría una verdadera retroalimentación, no solo sobre el cumplimiento del mandato, la suficiencia de los recursos y el progreso de las acciones, sino también sobre la pertinencia de prioridades y objetivos, la eficacia y eficiencia de las intervenciones y la capacidad de respuesta del Gobierno nacional a la problemática de la seguridad y salud de los trabajadores colombianos.

Ahora bien, el país se encuentra en el momento preciso para evaluar el impacto de la política pública y los programas nacionales, no solo con respecto a la gestión sistemática de los riesgos del trabajo y el control de la morbimortalidad laboral, sino con la perspectiva de su aporte a la calidad de vida, la producción empresarial, el cuidado del ambiente y el desarrollo sostenible global.

\section{Referencias bibliográficas}

1.Tomasina F. Los problemas en el mundo del trabajo y su impacto en salud. Crisis financiera actual. Revista de Salud Pública. 2012;14(1 Sup): 56-67.

2. Organización Mundial de la Salud (OMS), Comité Mixto OIT/OMS sobre Higiene del Trabajo. Tercer informe. Serie de Informes Técnicos N.o 135. Ginebra: OMS; 1957.

3. Betancourt O. Texto para la enseñanza e investigación de la salud y seguridad en el trabajo. Quito: OPS/OMSFUNSAD; 1999. 
4. GOHNET. Boletín GOHNET N.o 5. Ginebra: GOHNET; 2003.

5. Organización Internacional del Trabajo (OIT). Sistema de Gestión de la SST: una herramienta para la mejora continua. Ginebra: OIT; 2011.

6. GOHNET. Boletín GOHNET N.o 6. Ginebra: GOHNET; 2003.

7. Organización de las Naciones Unidas (ONU). 65 Asamblea General de Naciones. Nueva York: ONU; 2010.

8. Roth-Deubel AN. Políticas públicas: formulación, implementación y evaluación. Bogotá: Ediciones Aurora; 2002.

9. Torres-Melo J. Introducción a las políticas públicas: Conceptos y herramientas desde la relación entre Estado y ciudadanía. Bogotá: IEMP Ediciones; 2013.

10. Velásquez R. Hacia una nueva definición del concepto política pública. Desafíos. 2009;20:149-87.

11. Roth-Deubel AN. Enfoques para el análisis de políticas públicas. Bogotá: Universidad Nacional de Colombia; 2010.

12. Organización Internacional del Trabajo (OIT). Convenio 155 sobre seguridad y salud de los trabajadores. Ginebra: OIT; 1981.

13. Organización Internacional del Trabajo (OIT). Protocolo 155 de 2002. Relativo al Convenio de seguridad y salud de los trabajadores. Ginebra: OIT; 2005.

14. Organización Internacional del Trabajo (OIT). Convenio 187. Marco promocional para la seguridad y salud en el trabajo. Ginebra: OIT; 2006.

15. Organización Internacional del Trabajo (OIT). Recomendación 197 de 2006.

16. Organización Internacional del Trabajo (OIT). El enfoque estratégico de la OIT en materia de Seguridad y Salud en el Trabajo. Módulo 1 del Plan de Formación sobre Desarrollo de un Programa Nacional de Seguridad y Salud en el Trabajo. Ginebra: OIT; 2013.

17. Organización Internacional del Trabajo (OIT). Crear una cultura de prevención en materia de seguridad y salud. Ginebra: ILO; 2013.

18. Castela JL. Biblioteca virtual de desarrollo sostenible y salud ambiental de la OPS [Internet]. 2002 [citado 2017 ago. 28]. Disponible en: https://www.bvsde.paho.org/bvsacd/cd65/introduccion.pdf

19. Organización Internacional del Trabajo (OIT). Introducción al Sistema Nacional de Seguridad y Salud en el Trabajo. Módulo 2 del Plan de Formación sobre Desarrollo de un Programa Nacional de Seguridad y Salud en el Trabajo. Santiago de Chile: OIT; 2013.

20. Organización Internacional del Trabajo (OIT). Perfilnacional de seguridad y salud en el trabajo y análisis de la situación nacional. Módulo 3 del Plan de Formación sobre Desarrollo de un Programa Nacional de Seguridad ySalud en el Trabajo. 2013. Santiago de Chile: OIT; 2013.

21. Organización Internacional del Trabajo (OIT). www.ilo.org [Internet]. 2007 [citado 2017 ago. 28]. Disponible en: https://www.ilo.org/wcmsp5/groups/public/---ed_protect/---protrav/---safework/documents /policy/wcms_212074.pdf

22. Organización Internacional del Trabajo (OIT). Programas nacionales de seguridad y salud en el trabajo. Módulo 4 del Plan de Formación sobre Desarrollo de un Programa Nacional de Seguridad y Salud en el Trabajo. 2013. Santiago de Chile: OIT; 2013. 
23. Organización Internacional del Trabajo (OIT). Estrategia global en materia de seguridad y salud en el trabajo. Ginebra: OIT; 2004.

24. Organización Internacional del Trabajo (OIT). Acerca de la OIT [Internet]. Ginebra: OIT; 2017 [citado 2017 jul. 20]. Disponible en: https://www.ilo.org/global/about-the-ilo/lang--es/index.htm

25. Organización Mundial de la Salud (OMS). Acerca de la OMS [Internet]. Ginebra: OMS; 2017 [citado 2017 jul. 20]. Disponible en: https://www.who.int/about/es/

26. Organización Mundial de la Salud (OMS). Entornos laborales saludables: fundamentos y modelo de la OMS. Contextualización, prácticas y literatura de soporte. Ginebra: OMS; 2010.

27. Somavía J. El trabajo decente: Una lucha por la dignidad humana. Publicación OIT. Santiago de Chile: OIT; 2014.

28. Santos C, Rodrigo F. La estrategia iberoamericana de seguridad y salud en el trabajo 2010-2013: una oportunidad para avanzar socialmente. Arch Prev Riesgos Labor. 2010;13(1):5-6.

29. Organización Internacional del Trabajo (OIT). [Internet]. Ginebra: OIT; 2017 [citado 2017 jul. 20]. Disponible en https://www.ilo.org/global/about-the-ilo/history/lang--es/index.html

30. Organización Internacional del Trabajo (OIT). Constitución de la OIT. [Internet]. Ginebra: OIT; 1919 [citado 2017 ago. 17]. Disponible en: https://www.ilo.org/dyn/normlex/es/f?p=1000:62:0:NO:62:P62_LIST_ENT RIE_ID:2453907:NO

31. Organización Internacional del Trabajo (OIT). Declaración de FiladelÆaFiladelÆa ILO; 1944.

32. Organización Internacional del Trabajo (OIT). Convenios y Recomendaciones. [Internet]. Ginebra: OIT; 2017 [citado 2017 ago. 17]. Disponible en: https://www.ilo.org/global/standards/introduction-to-international-lab our-standards/conventions-and-recommendations/lang--es/index.htm

33. Organización Internacional del Trabajo (OIT). Normlex. RatiÆaciones de Colombia. [Internet] [citado 2018 mayo 18]. Disponible en: https://www.ilo.org/dyn/normlex/es/f?p=NORMLEXPUB:11200:0:NO:P11200_ COUNTRY_ID:102595

34. Gómez N, Turizo F. Seguridad y salud en el trabajo en Colombia: retos frente a las personas con discapacidad. Revista CES Derecho. 2016;7(2):84-94.

35. Ministerio de Trabajo, Alcaldía Mayor de Bogotá, et al. Política Pública de Trabajo Decente y Digno. Documento síntesis. Bogotá: Alcaldía; 2015.

36. Ghai D. Trabajo Decente. Concepto e indicadores. Revista Internacional del Trabajo. 2003;122(2):125-60.

37. Organización Internacional del Trabajo (OIT). Directrices relativas a los sistemas de gestión de la seguridad y salud en el trabajo ILO - OSH 2001. Ginebra: OIT; 2001.

38. Organización Internacional del Trabajo (OIT). Historia del 28 de abril. Página web. Ginebra: OIT; 2017.

39. OIT, ISSA, KOSHA. Declaración de Seúl sobre seguridad y salud en el trabajo [Internet]. Seúl: OIT; 2008. Disponible en: https://www.ilo.org/wcmsp5/groups/public/@dgreports/@dcomm/documents/statement/w cms_095955.pdf

40. Organización de las Naciones Unidas (ONU). Objetivos de Desarrollo Sostenible 2015-2030. Nueva York: ONU; 2015.

41. Organización Internacional del Trabajo (OIT). www.ilo.org. [Internet]; 2017 [citado 2017 sept. 9]. Disponible en: https://www.ilo.org/safework/events/safeday/lang--es/index.htm

42. Organización Panamericana de la Salud (OPS). Historia de la OPS. Washington: OPS; 2017.

43. Organización Panamericana de la Salud (OPS). Acerca de la OPS [Internet]; Washington: OPS; 2017. Disponible en: https://www.paho.org/hq/index.php?option=com_content\&view=article\&id=91:about-paho \&Itemid $=220 \&$ lang $=$ es 
44. Organización Mundial de la Salud (OMS). Constitución Organización Mundial de la Salud con reformas [Internet]. Ginebra: OMS; 2006. Disponible en: https://www.who.int/governance/eb/who_constitution_sp. pdf

45. Organización Panamericana de la Salud (OPS). Declaración de Alma Ata. Alma Ata: OPS; 1978.

46. Organización Mundial de la Salud (OMS), Organización Panamericana de la Salud (OPS). Estrategia Mundial de la Salud Ocupacional para todos. Ginebra: OMS, OPS; 1995.

47. Organización Panamericana de la Salud (OPS). Relatoría Taller Estrategia de Promoción de la Salud en los lugares de trabajo de América Latina y el Caribe. San José de Costa Rica: Organización Panamericana de la Salud, Organización Mundial de la Salud División de Salud y Ambiente Programa Regional de Salud de los Trabajadores, Red de Empresas, Centros y Puestos de Trabajo Saludables HeCONET; 2000.

48. Organización Panamericana de la Salud (OPS). Plan Regional de Salud de los Trabajadores. Washington: OPS; 2001.

49. Organización Mundial de la Salud (OMS). Resultados de la cumbre mundial sobre el desarrollo sostenible e implicaciones para el seguimiento. Consejo Ejecutivo EB111/31 reunión 111 punto 10.4 del orden del día 12 de dic de 2002. Johannesburgo-Suráfrica, 2002.

50. Organización Mundial de la Salud (OMS). Carta de Bangkok. Bangkok: OMS; 2005.

51. Organización Mundial de la Salud (OMS). Declaración de Stresa - Declaración de Salud de los trabajadores. Stresa: OMS; 2006.

52. Organización Mundial de la Salud (OMS). Salud de los Trabajadores: Plan de Acción Mundial. Ginebra: OMS; 2007.

53. Organización Panamericana de la Salud (OPS) - Organización Mundial de la Salud (OMS). Plan de acción sobre la salud de los trabajadores 2015-2025. [Internet] [citado 2018 mayo 18]. Disponible en: https://iris.paho.org/ xmlui/bitstream/handle/123456789/33983/CD54_10Rev.\%201-spa.pdf?sequence=1\&isAllowed=y

54. Organización Internacional del Trabajo (OIT). www.ilo.org. [Internet]. 2013 [citado 2017 sept. 17]. Disponible en: https://www.ilo.org/wcmsp5/groups/public/---americas/---ro-lima/documents/publication/ wcms_214985.pdf

55. Unión Europea (UE). Estrategia Comunitaria SST 2002-2006. Lisboa: UE; 2004.

56. Unión Europea (UE). Comunicación relativa a un marco estratégico de la UE en materia de salud y seguridad en el trabajo. Bruselas: UE; 2014.

57. Consejo Sub-Regional de Trabajadores Andinos. La situación socio-laboral en la subregión andina. Bogotá: 2008. https://www.ituc-csi.org/IMG/pdf/La_situacion_socio-laboral_en_la_subregion_andina.pdf

58. Comunidad Andina de Naciones (CAN). Instrumento Andino de Seguridad y Salud en el trabajo. En: Decisión 584 de la Comunidad Andina de Naciones. Guayaquil: CAN; 2004.

59. Organización de las Naciones Unidas (ONU). Declaración Universal de los Derechos Humanos. En Asamblea General de las Naciones Unidas - Resolución 217 A (III); París: ONU; 1948.

60. Santos-García CJ. La Organización Iberoamericana de Seguridad Social. Med Segur Trab. 2007;53(209):25-31.

61. OISS. I Estrategia Iberoamericana de Seguridad y Salud en el Trabajo. Madrid: OISS; 2010.

62. OISS. II Estrategia Iberoamericana de Seguridad y Salud en el Trabajo 2015-2020. Madrid: OISS; 2015.

63. Riaño-Casallas MI, Palencia-Sánchez F. Dimensión económica de la seguridad y salud en el trabajo: una revisión de literatura. Revista Gestión y Políticas de Salud. 2016;15(30):24-37.

64. Rodríguez-Villamizar LA, et al. Necesidades de investigación y formación en salud ambiental y ocupacional en Colombia: Un estudio Delphi. Biomédica. 2015;35: 24-30.

65. Presidencia de la República de Colombia. Constitución Política de 1886 Asamblea Nacional Constituyente.www.alcaldiabogota.gov.co. [Internet]. Bogotá; 1886 [citado 20 Jūl. 24]. Disponible en: https ://www.alcaldiabogota.gov.co/sisjur/normas/Norma1.jsp?i=7153 
66. Arenas-Monsalve G. Los riesgos de trabajo y la salud ocupacional en Colombia. Segunda edición. Bogotá: Legis; 1991.

67. Organización Internacional del Trabajo (OIT). www.ilo.org [Internet]. Lima; 2017 [citado 2017 jul. 24]. Disponible en: https://www.ilo.org/lima/paises/colombia/lang--es/index.htm

67. Congreso de la República de Colombia. Ley 6 de 1945. Por la cual se dictan algunas disposiciones sobre convenciones de trabajo, asociaciones profesionales, conflictos colectivos y jurisdicción especial de trabajo. Diario Oficial 25790.

69. República de Colombia. Ministerios de Trabajo y de Higiene. Decreto 3767 de 1949. Por el cual se reglamenta Ley 77 de 1948; el funcionamiento de la Oficina Nacional de Medicina e Higiene Industrial del Ministerio delTrabajo; el inciso $2^{\circ}$ del artículo $8^{\circ}$ de la Ley 95 de 1946; el artículo $9^{\circ}$ del Decreto-Ley 320 de 1949, y se dictan otras disposiciones.www.suin-juriscol.gov.co. [Internet]; 1949 [citado 2017 jul. 24]. Disponible en: https://ww w.suin-juriscol.gov.co/viewDocument.asp?id=1536392

70. Arenas-Monsalve G. Derecho colombiano de la seguridad social. Tercera edición. Bogotá: Legis; 2011.

71. OIT, NORMALEX. Ratificaciones de Colombia. OIT. Disponible en: https://www.ilo.org/dyn/normlex/es/f? $\mathrm{p}=1000: 11200: 0::$ NO:11200:P11200_COUNTRY_ID:102595

72. Organización de las Naciones Unidas (ONU). Oficina Alta Comisionada de DDHH. www.ohchr.org. [Internet]; 1966 [citado 2017 jul. 24]. Disponible en: https://www.ohchr.org/

73. Congreso de la República. Ley 9 de 1979. Por la cual se dictan medidas sanitarias. Diario Oficial N.o 35308 del 16 de julio de 1979.

74. República de Colombia. Ministerio de Trabajo y Seguridad Social. www.ilo.org. [Internet]; 1979 [citado 2017 jul. 24]. Disponible en: www.ilo.org/dyn/legosh/en/f?p=LEGPOL:503:785265035592:503:P503

75. República de Colombia. Presidencia. Plan de Integración Nacional 1979 - 1982. cdim.esap.edu.co [Internet]; 1979 [citado 2017 jul. 24]. Disponible en: https://www.cdim.esap.edu.co/BancoMedios/Documentos\% d_plan\%20nacional\%20de\%20desarrollo_cesar\%20turbay_1978_1982.pdf

76. García AC. El sistema general de riesgos profesionales vigente en Colombia. Revista Colombiana de Derecho Internacional. 2008;(13):215-53.

77. Presidencia de la República, Ministerios de Trabajo y Seguridad Social, de Salud y de Educación. Decreto 614 de 1984. Por el cual se determinan las bases para la organización y administración de Salud Ocupacional en el país. Diario Oficial 36561 del 14 de marzo de 1984.

78. Ministerios de Trabajo y Seguridad Social. Resolución Conjunta 1016 de 1989. Por la cual se reglamenta la organización, funcionamiento y forma de los Programas de Salud Ocupacional que deben desarrollar los patronos o empleadores en el país.

79. Ministerio de Trabajo. Resolución 2013 de 1986. Por la cual se reglamenta la organización y funcionamiento de los Comités de Medicina, Higiene y Seguridad Industrial en los lugares de trabajo.

80. Ministerio de Trabajo y Seguridad Social. Decreto 778 de 1987. Por el cual se modifica la tabla de enfermedades profesionales contenida en el artículo 201 del Código Sustantivo del Trabajo. Diario Oficial N.o 37.868.

81. Ministerios de Trabajo, Salud e ISS. Plan Nacional de Salud Ocupacional 1990-1995. Bogotá: Ministerio de Trabajo y Seguridad Social y de Salud, Instituto de Seguros Sociales, Comité Nacional de Salud Ocupacional; 1990-1995.

82. Presidencia de la República de Colombia. www.alcaldiabogota.gov.co. [Internet]. Bogotá: Ministerios de Hacienda y Crédito Público, de Trabajo y Seguridad Social, Salud; 1994 [citado 2017 jul. 24]. Disponible en: http://www.alcaldiabogota.gov.co/sisjur/normas/Norma1.jsp?i=16752. 
83. Ministerio de Salud. www.minsalud.gov.co [Internet]; 2017 [citado 2017 jul. 24]. Disponible en: https://www. minsalud.gov.co/Lists/FAQ/DispForm.aspx?ID=836

84. Ministerio de la Protección Social y Fondo de Riesgos Profesionales. Plan Nacional de Salud Ocupacional 2008-2012. Bogotá: Ministerio de la Protección Social y Fondo de Riesgos Profesionales; 2009.

85. OISS-CIFOISS. Evaluación al seguimiento de la implementación de los indicadores del PNSST. Presentación técnica. Bogotá: OISS-CIFOISS; 2015.

86. Congreso de la República de Colombia. Ley 776 de 2002. Por la cual se dictan normas sobre la organización, administración y prestaciones del Sistema General de Riesgos Profesionales. Diario Oficial 45037 de diciembre 17 del 2002.

87. Ministerio de la Protección Social. Informe de enfermedad profesional en Colombia 2000-2002. Una oportunidad para la prevención. Bogotá: Ministerio de la Protección Social; 2004.

88. Ministerio Protección Social. Tercer Plan Nacional de Salud Ocupacional 2003-2007. Bogotá: Ministerio de la Protección Social; 2003.

89. Ministerio de la Protección Social. Resolución 156 de 2005, por la cual se adoptan los formatos de informe de accidente de trabajo y de enfermedad profesional y se dictan otras disposiciones. Diario Oficial 45809 de febrero 1 del 2005.

90. Ministerio de la Protección Social. Resolución 2844 del 2007. Por la cual se adoptan las guías de atención integral de salud ocupacional basadas en la evidencia.

91. Ministerio de la Protección Social. Resolución 1013 de 2008. Por la cual se adoptan las guías de atención integral de salud ocupacional basadas en la evidencia para asma ocupacional, trabajadores expuestos a Benceno, plaguicidas inhibidores de la colinesterasa, dermatitis de contacto y cáncer pulmonar relacionados con el trabajo. Diario Oficial 46943 del 2008

92. Ministerio de la Protección Social. Resolución 2346 por la cual se regula la práctica de evaluaciones médicas ocupacionales y el manejo y contenido de las historias clínicas ocupacionales. Oficial 46691 del 16 de julio del 2007.

93. Ministerio de la Protección Social. Resolución 1918 del 2009. Modifica los artículos 11 y 17 de la Resolución 2346 de 2007 y se dictan otras disposiciones. Diario Oficial 47.377 del 11 de junio del 2009.

94. Ministerio de la Protección Social. Resolución 1401 de 2007. Por la cual se reglamenta la investigación de incidentes y accidentes de trabajo. Diario Oficial 46638 del 24 de mayo del 2007.

95. Ministerio de la Protección Social, SENA. diagnóstico actual y prospectivo de la salud ocupacional y los riesgos laborales con enfoque de entornos. Bogotá: Ministerio de la Protección Social, SENA; 2005.

96. Ministerio de la Protección Social. Primera Encuesta Nacional de Condiciones de Salud y Trabajo en el Sistema General de Riesgos Laborales. Reporte. Bogotá: Ministerio de la Protección Social; 2007.

97. Organización Internacional del Trabajo. www.ilo.org. [Internet]; 2006 [citado 2017 sept. 9]. Disponible en: http s://staging.ilo.org/public/libdoc/ilo/2006/106B09_56_span.pdf

98. Ministerio Protección Social. Cuarto Plan Nacional de Salud Ocupacional 2008-2012; Bogotá: Ministerio de la Protección Social; 2008.

99. Ministerio de la Protección Social. Por el cual se adopta el Plan Nacional de Salud Pública 2007-2010. Bogotá. Diario Oficial 46716 del 10 de agosto del 2007.

100. Ministerio de la Protección Social. Decreto 2566 de 2009. Por el cual se adopta la Tabla de Enfermedades Profesionales. Diario Oficial 47.404 del 8 de julio del 2009.

101. Ministerio de la Protección Social. Resolución 3673 del 2008. Por la cual se establece el Reglamento Técnico de Trabajo Seguro en Alturas. 
102. Ministerio de la Protección Social. Decreto 2923 de 2011. Por el cual se establece el Sistema de Garantía de Calidad del Sistema General de Riesgos Profesionales. Diario Oficial 48163 del 16 de agosto del 2011.

103. Ministerio de Trabajo. Informe ejecutivo de la segunda encuesta nacional de condiciones de seguridad y salud en el trabajo en el Sistema General de Riesgos Laborales en Colombia. Bogotá: Ministerio de Trabajo; 2013.

104. Congreso de la República de Colombia. Ley 1562 de 2012. Por la cual se modifica el Sistema de Riesgos Laborales y se dictan otras disposiciones en materia de Salud Ocupacional. Diario Oficial N.o 48.488 del 11 de del 2012.

105. Ministerio del Trabajo. Resolución 6045 del 2014. Por la cual se adopta el Plan Nacional de Seguridad y Salud en el Trabajo 2013-2021L. Diario Oficial N.o 49969 del 18 de agosto del 2016.

106. Ministerio de Trabajo. Plan Nacional de Seguridad y Salud en el Trabajo 2013-2021. Bogotá: Ministerio de Trabajo; 2013.

107. Zambrano AM. Capítulo 1. Dinámica del mercado laboral en Colombia. En: Análisis del mercado laboral en Colombia: una nueva cara de la formalidad. Reporte. Bogotá: Fasecolda; 2015.

108. Ministerio de Salud y Protección Social. Plan Decenal de Salud Pública 2012-2021. Bogotá: Ministerio de Salud y Protección Social; 2013.

109. Congreso de la República de Colombia. Ley 1753 de 2015. Por la cual se expide el Plan Nacional de Desarrollo 2014-2018 “Todos por un nuevo país”. Diario Oficial N.o 49.538 del 9 de junio del 2015.

110. Presidencia de la República. Decreto 2362. Día del Trabajo Decente en Colombia. Diario Oficial N.o 49719 del 7 de diciembre del 2015.

\section{Licencia Creative Commons CC BY 4.0}

Para citar este artículo: Álvarez Torres SH, Riaño-Casallas MI. La política pública de seguridad y salud en el trabajo: el caso colombiano. Rev. Gerenc. Polit. Salud. 2018;17(35). https://doi.org/10.11144/ Javeriana.rgps17-35.ppss 